June 1993

LBL-33777, UCB-PTH-93/08

KUL-TF-93/25

hep-th/9306135

\title{
Regularisation of non-local actions in two-dimensional field theories.
}

\author{
Alexander Sevrin ${ }^{a}$, Ruud Siebelink $\left.\right|^{\mathbb{p}}$ and Walter Troost $\left.\right|^{b}$ \\ a. Department of Physics \\ University of California at Berkeley \\ and \\ Theoretical Physics Group \\ Lawrence Berkeley Laboratory \\ Berkeley, CA 94720, U.S.A. \\ b. Instituut voor Theoretische Fysica \\ Universiteit Leuven \\ Celestijnenlaan 200D, B-3001 Leuven, Belgium
}

\begin{abstract}
Taking the induced action for gauge fields coupled to affine currents as an example, we show how loop calculations in non-local two-dimensional field theories can be regulated. Our regularisation method for one loop is based on the method of Pauli and Villars. We use it to calculate the renormalisation factors for the corresponding effective actions, clearing up some discrepancies in the literature. In particular, it will be shown explicitly that vector gauge transformation invariance and Haar invariance of functional integral measures impose different requirements, but they are related by a counterterm (which is local in terms of group variables). For higher loops, we use the method of covariant derivatives combined with Pauli-Villars to argue that the one loop result remains unaltered.
\end{abstract}

\footnotetext{
${ }^{1}$ Aspirant NFWO, Belgium

${ }^{2}$ Bevoegdverklaard Navorser NFWO, Belgium
} 


\section{Disclaimer}

This document was prepared as an account of work sponsored by the United States Government. Neither the United States Government nor any agency thereof, nor The Regents of the University of California, nor any of their employees, makes any warranty, express or implied, or assumes any legal liability or responsibility for the accuracy, completeness, or usefulness of any information, apparatus, product, or process disclosed, or represents that its use would not infringe privately owned rights. Reference herein to any specific commercial products process, or service by its trade name, trademark, manufacturer, or otherwise, does not necessarily constitute or imply its endorsement, recommendation, or favoring by the United States Government or any agency thereof, or The Regents of the University of California. The views and opinions of authors expressed herein do not necessarily state or reflect those of the United States Government or any agency thereof of The Regents of the University of California and shall not be used for advertising or product endorsement purposes.

Lawrence Berkeley Laboratory is an equal opportunity employer. 


\section{Introduction}

In two-dimensional field theory, the chiral algebra that constitutes (part of) the symmetry algebra is of central interest. Classically, coupling gauge fields to that current algebra introduces a local invariance, which in turn can be used to gauge away the gauge fields, so that these do not acquire a dynamical existence. Quantum mechanically however, due to anomalies, these gauge fields often do become propagating fields, describing new degrees of freedom. The best known case is of course the Liouville mode for non-critical strings. The action used as a starting point to describe the quantum theory of these new degrees of freedom is the induced action, i.e. the action resulting from integrating out the 'matter' degrees of freedom that make up the chiral currents. In the case of linear chiral algebras, where the central charge $c$ only appears in the most singular term of the operator product expansions, this induced action is proportional to $c$. For non-linear algebras, the central charge also appears elsewhere in coupling constants, and the induced action can be expanded in

a power series, $\Gamma_{\text {ind }}=\sum_{i=0}^{\infty} c^{1-i} \Gamma^{i}$ where $\Gamma^{0}$ will be called the 'classical' term. Building a quantum theory on this induced action, the following remarkable renormalisation property has been noticed: the quantum effective action has the same functional form as the classical term in the induced action, but a renormalisation of fields and couplings takes place :

$$
\Gamma_{\text {eff }}[\Phi]=Z_{\Gamma} \Gamma^{0}\left[Z_{\Phi} \Phi\right]
$$

This property has been verified for Wess-Zumino-Witten models [1] in [2], for twodimensional gravity in [3], for $W_{3}$ gravity in [4] to first order and to all orders in [5] It was recently extended [6], also to all orders, to all extensions of two-dimensional gravity that can be obtained from a Drinfeld-Sokolov reduction of WZW models based on (super)algebras [7].

The WZW models are the simplest examples, and in view of the Drinfeld-Sokolov reduction technique, at the same time also generic. For these models, there actually exist several different methods to obtain eq.(1). We will recapitulate the different methods in the next section. These methods agree as to the general form of the result, and also as to the value of $Z_{\Gamma}$. However, they disagree on the field renormalisation factors $Z_{\Phi}$. In view of the universal applicability of eq.(11), valid as it seems to be for a very wide class of chiral algebras, this is somewhat disconcerting. As will become clear, each of these methods can be criticized on the grounds that little attention is payed to one of the prominent features of quantum field theory, namely the need to make sense of the divergent expressions as they are encountered in perturbation theory. Partly this is due to the fact that sometimes divergent diagrams, when treated in a cavalier fashion, formally lead to reasonable finite expressions, which are then taken to be the final answer. However, certainly since the advent of anomalies, we have learned that even these finite parts may turn out to have a value that is different from the 'naive' one. Perhaps the simplest example is the box diagram contribution to photon-photon scattering in QED: gauge invariance requires it to vanish at zero momenta, but a simple four-dimensional calculation 
leads to a finite integral that is non-zero. Needless to say a regularised version (whether Pauli-Villars, dimensional regularisation, or others) leads to the correct (zero) value. It will be shown in this paper that a similar phenomenon occurs for the (finite) renormalisation factors for induced two-dimensional gauge theories.

In order to resolve the discrepancy then, one has to face the issue of regularisation in non-local field theories. Indeed, the induced actions are typically non-local. In local field theory, one has developed by now a whole arsenal of techniques to deal with the divergences that occur in a perturbative expansion of Green functions (or $S$-matrix elements). They typically involve the introduction of a cut-off at high momenta. Then some renormalisation conditions are imposed, defining the physical parameters of the theory in terms of the parameters in the Lagrangian as well as the cut-off. Finally, the cut-off is taken to infinity keeping the physical parameters equal to their experimental values. Alternatively, one introduces the physical parameters in the Lagrangian from the start, and adds counterterms to it, order by order in perturbation theory, to keep these parameters at their physical values. A crucial role is played in this whole development by the locality properties: all counterterms added, finite or infinite (i.e. terms with or without limit when the cut-off is removed), are local expressions in terms of the fields. In non-local field theories one loses this important criterium. We will take a very pragmatic attitude here, and first look for a method that regularises the theory. Rather than investigating all possible counterterms that one may reasonably (want to) add, we will at first not add any when none is needed. In this paper we will also limit ourselves to very specific non-local field theory, viz. the two-dimensional actions, induced by matter, of chiral gauge fields that couple to matter currents forming an affine Lie algebra. After introducing the model in section two, we will present three derivations of the renormalisation formula eq.(1) in section three. In the next section, based on simple considerations of the diagrams to be computed for the two point function, we will introduce a regularisation for the quantum theory, including single loops only. This regularisation can be translated to a Pauli-Villars style regularisation [8], which will have the unusual property that it is based on a nonlocal mass term. Then we will go on to show that it regularises all one-loop diagrams, and we will compute the $n$-point functions in the same 1-loop approximation. In section six we will compare the resulting renormalisation factors with the formal calculations of the third section. Also in that section we will relate our computation to computations in the WZW model, which is closer to a local field theory than the induced actions for gauge fields we consider up to there. We will show that the regularisation we adopted is fully compatible with the formal Haar-invariance of the WZW integration measure, provided one includes a finite local counterterm. In section seven we propose a regularisation method that goes beyond one loop, introducing a method based on higher derivative terms in the Lagrangian (which are introduced covariantly), followed by a Pauli-Villars style regularisation of the remaining one-loop divergences. We will show that the one-loop result obtained in section five remains unaltered, and that it in fact provides the full answer. 


\section{Induced Wess-Zumino-Witten models}

The induced action $\Gamma[A]$ for a Lie algebra valued gauge field $A$ is defined to be the generating functional of all connected current-current correlators for a given holomorphic affine Lie algebra of level $k$ : ?

$$
e^{-\Gamma[A]}=\left\langle e^{-\frac{1}{\pi x} \int d^{2} z \operatorname{tr}\{J(z) A(z)\}}\right\rangle
$$

and correspondingly, for an anti-holomorphic algebra one defines the induced action $\bar{\Gamma}[\bar{A}]$ for a gauge field of opposite chirality. These induced actions arise when integrating out 'matter' degrees of freedom, indicated by the \langle\rangle signs. All that is needed from this underlying model are the operator product expansions for the currents $J^{a}$, which we take to form an affine Lie algebra. Explicitly,

$$
\begin{aligned}
\Gamma[A]= & \frac{k}{2 \pi x} \int d^{2} z \operatorname{tr}\left\{\frac{1}{2} A \frac{\partial}{\bar{\partial}} A-\frac{1}{3} \frac{1}{\bar{\partial}} A\left[A, \frac{\partial}{\bar{\partial}} A\right]\right. \\
& \left.-\frac{1}{4} \frac{1}{\bar{\partial}} A\left[A, \frac{1}{\bar{\partial}}\left(\left[A, \frac{\partial}{\bar{\partial}} A\right]\right)\right]+\cdots\right\} .
\end{aligned}
$$

Although this action is non-local, its variation under the gauge transformation

$$
\delta_{\eta} A=\bar{\partial} \eta-[A, \eta]=\bar{D}[A] \eta
$$

is a purely local expression, given by

$$
\delta_{\eta} \Gamma[A]=-\frac{k}{2 \pi x} \int d^{2} z \operatorname{tr}\{\eta \partial A\} .
$$

This transformation property in fact contains all the information needed concerning the induced action. Introducing the "induced current" $u$

$$
u_{a}(x)=-2 \pi \frac{\delta \Gamma[A]}{\delta A^{a}(x)}
$$

one finds from eq. (5) the Ward identity

$$
\bar{D}[A] u / k=\partial A .
$$

\footnotetext{
${ }^{3}$ We use the following conventions: If $\left[t_{a}, t_{b}\right]=f_{a b}{ }^{c} t_{c}$, then $f_{a c}{ }^{d} f_{b d}{ }^{c}=-\tilde{h} g_{a b}$, where $\tilde{h}$ is the dual Coxeter number. In a representation $R$ we have $\operatorname{tr}\left\{t_{a} t_{b}\right\}=-x g_{a b}$, with $x$ the index of the representation
} 
In [9 it was realised that this Ward identity is nothing but the statement that the curvature for the Yang-Mills field with components $\{A, u / k\}$ vanishes. This condition is then solved by parametrizing $A \equiv \bar{\partial} g g^{-1}$ and $u / k \equiv \partial g g^{-1}$. Rewriting the righthand side of eq. (5) using $A=\bar{\partial} g g^{-1}$ and $\eta=\delta_{\eta} g g^{-1}$ one obtains the equation of motion of a Wess-Zumino-Witten model. One concludes that

$$
\Gamma\left[A=\bar{\partial} g g^{-1}\right]=-k S^{+}[g]
$$

with

$$
S^{+}[g]=\frac{1}{4 \pi x} \int d^{2} z \operatorname{tr}\left\{\partial g^{-1} \bar{\partial} g\right\}+\frac{1}{12 \pi x} \int d^{3} x \epsilon^{\alpha \beta \gamma} \operatorname{tr}\left\{g_{, \alpha} g^{-1} g_{, \beta} g^{-1} g_{, \gamma} g^{-1}\right\}
$$

Having established a dynamical, though non-local, action for the gauge field $A$, one proceeds with the subsequent quantisation of this theory. The objects of interest are the generating functionals $Z[u]$ and $W[u]$ of the Green functions of this quantum theory:

$$
Z[u]=e^{-W[u]}=\int \mathcal{D} A e^{-\Gamma[A]+\frac{1}{2 \pi x} \int \operatorname{tr}\{u A\}},
$$

or, equivalently, the Legendre transform $\Gamma$ :

$$
\Gamma\left[A_{\mathrm{cl}}\right]=\min _{\{u\}}\left(W[u]-\frac{1}{2 \pi x} \int \operatorname{tr}\left\{u A_{\mathrm{cl}}\right\}\right) .
$$

which is the generating functional for one particle irreducible diagrams, and is called the effective action. For brevity, we will use the same name for $W[u]$ also. In the sequel we will also use the level-independent reference functional $\Gamma^{0}[A]=\Gamma[A] / k$ and its Legendre transform

$$
W^{0}[u]=\min _{\{A\}}\left(\Gamma^{0}[A]-\frac{1}{2 \pi x} \int \operatorname{tr}\{u A\}\right)
$$

The explicit form of this last quantity is given by

$$
W^{0}\left[u \equiv \partial g g^{-1}\right]=S^{+}\left[g^{-1}\right]
$$

The opposite chirality can be treated along the same lines. We have that $\bar{\Gamma}^{0}[\bar{A}=$ $\left.\partial \bar{g} \bar{g}^{-1}\right]=-S^{-}[\bar{g}]$. Because of the identity $S^{-}[\bar{g}]=S^{+}\left[\bar{g}^{-1}\right]$, we have also that

$$
W^{0}[\bar{A}]=-\bar{\Gamma}^{0}[\bar{A}]
$$




\section{Three Derivations of the Effective Action}

In this section we review three different methods to calculate the effective action $W[u]$.

The first method is a semiclassical approximation [10, 11]. According to the steepest descent method the effective action eq.(10) is approximated by

$$
e^{-W[u]} \simeq e^{-\Gamma\left[A_{\mathrm{cl}}\right]+\frac{1}{2 \pi x} \int \operatorname{tr}\left\{u A_{\mathrm{cl}}\right\}} \int \mathcal{D} \alpha e^{-\frac{1}{2} \int \alpha^{a} \frac{\delta^{2} \Gamma^{0}\left[A_{\mathrm{cl}}\right]}{\delta A_{\mathrm{cl}}^{a} \delta A_{\mathrm{cl}}^{b}} \alpha^{b}}
$$

where $A_{\mathrm{cl}}[u]$ is the saddle point and $\alpha$ is the fluctuation around this point. The position of the saddle is obtained by solving

$$
-2 \pi \frac{\delta \Gamma^{0}\left[A_{\mathrm{cl}}\right]}{\delta A_{\mathrm{cl}}^{a}}=\frac{u_{a}}{k}
$$

giving $A_{\mathrm{cl}}$ as a functional of $u$. Then, all that has to be done is to compute the functional determinant:

$$
W[u] \simeq k W^{0}[u / k]+\frac{1}{2} \log \operatorname{det} \frac{\delta^{2} \Gamma\left[A_{\mathrm{cl}}\right]}{\delta A_{\mathrm{cl}} \delta A_{\mathrm{cl}}} .
$$

To evaluate this expression we take advantage of the Ward identity. Indeed, taking the functional derivative of eq. (7) with respect to $A_{\mathrm{cl}}$, one obtains the operator identity

$$
-2 \pi \bar{D}_{x}\left[A_{\mathrm{cl}}\right]^{a c} \frac{\delta^{2} \Gamma^{0}\left[A_{\mathrm{cl}}\right]}{\delta A_{\mathrm{cl}}^{c}(x) \delta A_{\mathrm{cl}}^{b}(y)}=D_{x}[u / k]_{. b}^{a} \delta^{2}(x-y),
$$

where $D[u / k]$ is the covariant derivative $\partial-u / k$, and the subscript $x$ denotes the coordinate the differential operator is acting on. It follows that the sought-after determinant is equal to the ratio of two determinants involving only ordinary covariant derivatives:

$$
\operatorname{det} \frac{\delta^{2} \Gamma^{0}\left[A_{\mathrm{cl}}\right]}{\delta A_{\mathrm{cl}} \delta A_{\mathrm{cl}}}=\frac{\operatorname{det} D[u / k]}{\operatorname{det} \bar{D}\left[A_{\mathrm{cl}}\right]}
$$

These determinants can then be computed [12] by writing them out as pathintegrals over $b c$ ghost-antighost systems. The ghostsystems realise an (anti-) holomorphic current algebra with level $k_{\text {ghost }}=2 \tilde{h}$. Therefore,

$$
\begin{aligned}
\log \operatorname{det} D[u / k] & =2 \tilde{h} W^{0}[u / k] \\
\log \operatorname{det} \bar{D}\left[A_{\mathrm{cl}}\right] & =-2 \tilde{h} \Gamma^{0}\left[A_{\mathrm{cl}}\right] \\
\frac{1}{2} \log \operatorname{det} \frac{\delta^{2} \Gamma^{0}\left[A_{\mathrm{cl}}\right]}{\delta A_{\mathrm{cl}} \delta A_{\mathrm{cl}}} & =2 \tilde{h} W^{0}[u / k]-\frac{\tilde{h}}{k} \int u_{a} \frac{\delta W^{0}[u / k]}{\delta u_{a} / k} .
\end{aligned}
$$


This gives the semiclassical result, valid through order $k^{0}$ :

$$
W[u]=(k+2 \tilde{h}) W^{0}\left[\frac{u}{k+\tilde{h}}\right] .
$$

The second method is called the KPZ [3] approach (we adapt the treatment in [11]). One starts from the following action, containing gauge fields of both chiralities, which is invariant under the transformation of $A$ as in eq. (4) and $\delta_{\eta} \bar{A}=D[\bar{A}] \eta$ :

$$
\Gamma[A]+\bar{\Gamma}[\bar{A}]-\frac{k}{2 \pi x} \int \operatorname{tr}\{A \bar{A}\}
$$

The invariance follows from eq.(5). This action is taken as a starting point for quantisation. Because of the invariance, gauge fixing is necessary. The gauge freedom is used to put the gauge field $\bar{A}$ equal to some fixed but arbitrary value $\hat{\bar{A}}$. Introducing the corresponding ghosts, one obtains the BRST-invariant action:

$$
\Gamma[A, \hat{\bar{A}}, b, c]=\Gamma[A]+\bar{\Gamma}[\hat{\bar{A}}]-\frac{k}{2 \pi x} \int \operatorname{tr}\{\hat{\bar{A}} A\}+\frac{1}{2 \pi} \int b D[\hat{\bar{A}}] c .
$$

We consider the partition function corresponding to this action,

$$
\tilde{Z}[\hat{\bar{A}}]=\int \mathcal{D} A \mathcal{D} b \mathcal{D} c e^{-\Gamma[A, \hat{\bar{A}}, b, c]} .
$$

The partition function is independent of the gauge choice - in this case this means independent of the function $\hat{\bar{A}}$ - and can be normalised to one. Expressing the term $\bar{\Gamma}[\hat{\bar{A}}]$ of the gauge fixed action as a pathintegral over a right-handed fermionic mattersystem with level $k$ one obtains

$$
\begin{aligned}
1=\tilde{Z}[\hat{\bar{A}}]= & \int \mathcal{D} A e^{-\Gamma[A]+\frac{k}{2 \pi x} \int \operatorname{tr}\{\hat{\bar{A}} A\}} \\
& \int \mathcal{D} b \mathcal{D} c \mathcal{D} \bar{\psi} e^{-\frac{1}{2 \pi} \int b D[\hat{\bar{A}}] c+\bar{\psi}^{t} D[\hat{\bar{A}}] \bar{\psi}} .
\end{aligned}
$$

The background field $\hat{\bar{A}}$ couples to a current that consists of three parts: matter $(\bar{\psi})$, ghost $(b c)$ and gauge system $(A)$ currents. The first two are antiholomorphic affine currents with central extensions $k$ and $2 \tilde{h}$ respectively. The $\hat{\bar{A}}$-independence then implies that the gauge part, $J_{\text {gauge }}=-\frac{k}{2} A$ also constitutes an anti-holomorphic affine algebra with a compensating value of the central charge, i.e. with level $-(k+2 \tilde{h})$. Therefore, combining eq.(10), eq.(25) and the anti-holomorpic counterpart of eq.(2) we obtain

$$
e^{-W[u=k \hat{\bar{A}}]}=\int \mathcal{D} A e^{-\Gamma[A]+\frac{k}{2 \pi x} \int \operatorname{tr}\{\hat{\bar{A}} A\}}
$$




$$
\begin{aligned}
& =\left\langle\exp -\frac{1}{\pi x} \int d^{2} x \operatorname{tr}\left\{\hat{\bar{A}} J_{\text {gauge }}\right\}\right\rangle \\
& =e^{-(-k-2 \tilde{h}) \bar{\Gamma}^{0}[\hat{\bar{A}}]}
\end{aligned}
$$

and using eq.(14),

$$
W[u]=(k+2 \tilde{h}) W^{0}\left[\frac{u}{k}\right]
$$

This ends the second derivation.

The third method [2] consists first of all in a change of variable from the gauge field $A$ to group variables $g$,

$$
A=\bar{\partial} g g^{-1}
$$

From $\delta_{\eta} A=\bar{D}[A] \eta=\bar{D}[A]\left(\delta_{\eta} g g^{-1}\right)$ one infers that the following jacobian is picked up :

$$
\begin{aligned}
\mathcal{D} A & =\mathcal{D} g \operatorname{det} \bar{D}\left[A=\bar{\partial} g g^{-1}\right] \\
& =\mathcal{D} g e^{2 \tilde{h} S^{+}[g]}
\end{aligned}
$$

Then from eq.(10),

$$
e^{-W[u]}=\int \mathcal{D} g e^{(k+2 \tilde{h}) S^{+}[g]+\frac{1}{2 \pi x} \int \operatorname{tr}\left\{u \bar{\partial} g g^{-1}\right\}} .
$$

If one parametrises the source $u \equiv(k+2 \tilde{h}) \partial h h^{-1}$ and one uses the PolyakovWiegmann identity [9],

$$
S^{+}\left[h^{-1} g\right]=S^{+}\left[h^{-1}\right]+S^{+}[g]+\frac{1}{2 \pi x} \int \operatorname{tr}\left\{\partial h h^{-1} \bar{\partial} g g^{-1}\right\}
$$

one finds

$$
e^{-W[u]}=e^{-(k+2 \tilde{h}) S^{+}\left[h^{-1}\right]} \int \mathcal{D} g e^{(\kappa+2 \tilde{h}) S^{+}\left[h^{-1} g\right]} .
$$

Using now the invariance of the Haar measure, this last pathintegral evaluates to a constant, so

$$
W[u]=(k+2 \tilde{h}) W^{0}\left[\frac{u}{k+2 \tilde{h}}\right] .
$$

Let us take stock. We have presented three methods to get information on the effective action, and obtained eqs.(21,27, 31). Three times we were led to the same functional dependence, viz. that the quantum effective action is a rescaling of the classical action. Three times also, the resulting renormalisation factor for the action, 
which can be seen to be a coupling constant renormalisation, has the same value, $k \rightarrow k+2 \tilde{h}$. However, the field renormalisation factor $Z_{u}^{-1}$ was three times different, $k \rightarrow k+n \tilde{h}$ with $n=1,0,2$ respectively. It is clear that, to solve this puzzle, more care is needed in the heuristic steps of the above methods. For that purpose, we will introduce in the next section a scheme that allows us to properly take into account the quantum field theory divergences implicitly present in the above derivations.

The coupling constant renormalisation factor, common to the three methods, is expected to be valid beyond the one loop approximation. The argument rests on the multiple valuedness of the WZW functional $S^{+}$. To have in eq.(10) a univalued $Z[u]$-functional, the prefactor of $W^{0}$ has to be an integer. Since higher loop quantum corrections will modify it with terms of order $1 / k$, they should be absent. Note that no similar argument can be given for the field renormalisation factors. Thus both the fact that they are different, and that they are closely related, is unexplained.

\section{Regularisation}

To investigate the quantum theory based on the induced action, one can follow the canonical methods used for non-local actions. One rewrites the action as a sum of monomials in the fields, eq.(3). The quadratic term determines the propagator, the higher order monomials the vertices. The non-locality manifests itself only in the fact that the propagator has an unfamiliar form, and the vertices contain inverse powers of momenta. To decide on a regularisation of the resulting diagrams is a different matter however. One method would be to try and convert the theory to a local one first. For the present case, this would involve the change of variables as in eq. (28), which is possibly a sensitive issue to start with. A more direct confrontation with the difficulties was made in [13], in the framework of two-dimensional gravity theories. There, each diagram was regularised, by hand, by inserting an exponential cut-off in the momentum integration. This procedure is not without difficulties, as discussed in [13], with dependence of the diagrams on routings of momenta.

The method that we are about to introduce will stick closely to traditional methods based on the ideas of Pauli and Villars. We will at first have a look at the simplest diagrams to be regulated. It will be shown that this can be done straightforwardly by a compensating diagram with a modified propagator. Then we determine which Lagrangian corresponds to this modification. We regard this as a crucial step to ensure consistency of our treatment. The regularisation of the remaining one-loop diagrams is then completely fixed.

To see what we need, we start by considering the two-point function. The momentumspace Feynman rules can be read off from

$$
\begin{gathered}
-(2 \pi)^{3} \frac{\delta^{2} \Gamma^{0}\left[A_{\mathrm{cl}}\right]}{\delta A_{\mathrm{cl}}^{a}(q) \delta A_{\mathrm{cl}}^{b}(r)} \equiv \Delta_{a b}(q, r)+\sum_{n=3}^{\infty} V_{a b}^{n}\left[A_{\mathrm{cl}}\right](q, r) \\
=g_{a b} \frac{\bar{q}}{q} \delta^{2}(q+r)
\end{gathered}
$$




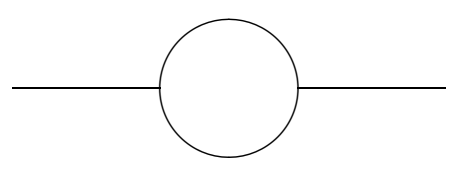

Figure 1: Vacuum Polarisation Diagram

$$
\begin{aligned}
& +\frac{2 i}{(2 \pi)^{2}} \int d^{2} p f_{a b c} A_{\mathrm{cl}}^{c}(p) \delta^{2}(p+q+r) \mathcal{V}_{3}(q, r, p) \\
& +\frac{1}{(2 \pi)^{4}} \int d^{2} p d^{2} s A_{\mathrm{cl}}^{d}(p) A_{\mathrm{cl}}^{e}(s) \delta^{2}(p+q+r+s) \\
& \quad\left\{f_{a d}^{c} f_{c e b} \mathcal{V}_{4}(q, r, p, s)+f_{a b}^{c} f_{c d e} \mathcal{W}_{4}(q, r, p, s)\right\} \\
& +\ldots \quad
\end{aligned}
$$

with

$$
\begin{aligned}
& \mathcal{V}_{3}(q, r, p)=\frac{p \bar{q}-\bar{p} q}{p q r} \\
& \mathcal{V}_{4}(q, r, p, s)=\frac{-1}{p+q}\left\{\frac{\bar{r}}{q r}-\frac{\bar{r}}{p r}+\frac{\bar{s}}{p s}-\frac{\bar{s}}{q s}+\frac{\bar{p}}{r p}-\frac{\bar{p}}{s p}+\frac{\bar{q}}{s q}-\frac{\bar{q}}{r q}\right\} \\
& \mathcal{W}_{4}(q, r, p, s)=\frac{-1}{p+s}\left\{\frac{\bar{r}}{p r}-\frac{\bar{s}}{q s}+\frac{\bar{s}}{r s}-\frac{\bar{q}}{p q}\right\}
\end{aligned}
$$

The two-point function at one loop order consists of the two diagrams of figs.1 and 2 .

The (unregularised) expression for the vacuum polarisation diagram (fig.1) is given by

$$
\begin{aligned}
& -\frac{1}{4} \operatorname{tr}\left\{\Delta^{-1} V^{3} \Delta^{-1} V^{3}\right\} \\
& =\frac{-\tilde{h}}{(2 \pi)^{4}} \int d^{2} p \int d^{2} q A_{\mathrm{cl}}^{a}(p) A_{\mathrm{cl} a}(-p) \frac{q^{2}}{|q|^{2}} \frac{(p+q)^{2}}{|p+q|^{2}} \frac{(\bar{p} q-\bar{q} p)^{2}}{q^{2}(p+q)^{2} p^{2}},
\end{aligned}
$$

which is logarithmically divergent. We refrain from making formal simplications before the integral is well-defined. To regularise it we add a supplementary diagram, with an identical integrand except for the replacement of the propagator denominators $|q|^{2}$ and $|p+q|^{2}$ by $|q|^{2}+M^{2}$ and $|p+q|^{2}+M^{2}$, and a change in overall sign. This improves the convergence by two powers which is sufficient in this case. We therefore adopt the following convergent expression for this diagram

$$
\sum_{i=0} c_{i} \frac{-\tilde{h}}{(2 \pi)^{4}} \int d^{2} p \int d^{2} q A_{\mathrm{cl}}^{a}(p) A_{\mathrm{cl} a}(-p) \frac{(\bar{p} q-\bar{q} p)^{2}}{p^{2}\left(|q|^{2}+M_{i}^{2}\right)\left(|p+q|^{2}+M_{i}^{2}\right)}
$$




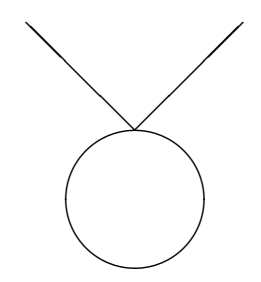

Figure 2: Seagull Diagram

where the sum runs over the original $i=0$ term (i.e. $c_{0}=1$ and $M_{0}=0$ ), and over (all) the Pauli-Villars term(s) needed to render the Feynman diagram well defined. We take $\sum_{i=0} c_{i}=0$. After the momentum integration, the limit $M_{i} \rightarrow \infty(i \neq 0)$ still has to be taken.

The Lagrangian interpretation for these regularising steps is quite simple. The extra contributions correspond to extra (PV) fields, having the same couplings as the original ones but different propagators, and an extra factor $c_{i}$ for the loop. This factor can be added by hand, or, perhaps more attractively, by limiting oneself to $c_{i}= \pm 1$ and considering -1 to result from the sum of two fermionic $(-2)$ and one bosonic $(+1) \mathrm{PV}$-field. The change in the propagator can be viewed as arising from a massterm for the PV-fields $\alpha_{i a}$

$$
\frac{-M_{i}^{2}}{16 \pi} \int \alpha_{i}^{a} \frac{1}{\bar{\partial}^{2}} \alpha_{i a}
$$

A non-local mass term has been used in a different context in [14]. If one substitutes $A=\bar{\partial} \varphi$ in eq.(3), the quadratic terms (and the trilinear terms) become local in $\varphi$, and the proposed mass term has a completely conventional form. We adopt it from now on.

The expression for the seagulldiagram (fig.2) is now completely fixed. One finds that

$$
\begin{aligned}
& \frac{1}{2} \sum_{i=0} c_{i} \operatorname{tr}\left\{\Delta_{i}^{-1} V^{4}\right\} \\
& =\sum_{i=0} c_{i} \frac{-\tilde{h}}{(2 \pi)^{4}} \int d^{2} p \int d^{2} q A_{\mathrm{cl}}^{a}(p) A_{\mathrm{cl} a}(-p) \frac{(\bar{p}+\bar{q})(p-q)(\bar{p} q-\bar{q} p)}{p^{2}\left(|q|^{2}+M_{i}^{2}\right)|p+q|^{2}} .
\end{aligned}
$$

Two remarks are in order. First, the linear divergence is again cured by the condition $\sum_{i=0} c_{i}=0$. Second, note that the vertex denominators are not altered, but only the propagators (for the PV contributions).

Both expressions (32) and (34) are thus made finite. It is convenient to combine them before taking the limit $M_{i} \rightarrow \infty$. This leads, after some elementary algebra, 
to the following formula for the one loop effective action:

$$
W_{1 \text { loop }}\left[u\left(A_{\mathrm{cl}}\right)\right]=\frac{-\tilde{h}}{(2 \pi)^{4}} \int d^{2} p A_{\mathrm{cl}}^{a}(p)\left(I_{4}+I_{33}\right) A_{\mathrm{cl} a}(-p)+\mathcal{O}\left(A_{\mathrm{cl}}^{3}\right)
$$

with

$$
I_{4}+I_{33}=\sum_{i=0} c_{i} \int d^{2} q \frac{(\bar{p} q-\bar{q} p)^{2}}{p^{2}\left(|q|^{2}+M_{i}^{2}\right)}\left\{\frac{1}{|p+q|^{2}+M_{i}^{2}}-\frac{1}{|p+q|^{2}}\right\}
$$

We want to stress here that the crucial difference in the two last denominators, is a direct consequence of the fact that the contributions from regulating fields are derived from a specific Lagrangian. As a result only propagators are modified in the PV-method, whereas vertices are simply duplicated, thus ensuring that all the oneloop diagrams of the original theory are regularised. The denominator coming from the non-local $V^{4}$-vertex therefore remains "massless". Formula (36) also indicates that the ad-hoc regularisation device of multiplying the integrand with a standard convergence factor, would give a vanishing result here.

To proceed with the evaluation of eq.(36) we combine the denominators using a Feynman parameter, shift the momenta and perform the momentum integral to find

$$
\begin{aligned}
I_{4}+\left.I_{33}\right|_{M_{i} \rightarrow \infty} & =\sum_{i} c_{i} \frac{-2 \pi|p|^{2}}{p^{2}} \int_{0}^{1} d \alpha \ln \frac{\alpha M_{i}^{2}+\alpha(1-\alpha)|p|^{2}}{M_{i}^{2}+\alpha(1-\alpha)|p|^{2}} \\
& =\frac{-2 \pi \bar{p}}{p}
\end{aligned}
$$

upon taking the limit. This limit might perhaps have contained divergent pieces (the individual contributions of the diagrams do). Then one would have had to adjust the action with some $\left(M_{i}\right.$-dependent) counterterms, and include their contribution before taking the limit. These counterterms are the ones that, in the usual renormalisation program, renormalise the physical vs. bare parameters in the Lagrangian. In the cases studied here, such divergent terms do not appear. Therefore, we don't

add any counterterms at present, and the infinite mass limit gives directly the final answer:

$$
W_{1 \text { loop }}\left[u\left(A_{\mathrm{cl}}\right)\right]=\frac{-2 \tilde{h}}{2 \pi x} \int d^{2} z \operatorname{tr}\left\{\frac{1}{2} A_{\mathrm{cl}} \frac{\partial}{\bar{\partial}} A_{\mathrm{cl}}+\cdots\right\}
$$

\section{One Loop Calculation of the Effective Action}

Having established our regularisation method by looking at the two-point function, we now compute the full effective action in the one loop approximation. In principle, this could be done with diagrams also, but we apply a simpler method. Let us consider the change in $W$ when we perform a gauge transformation on its argument, $\delta(u / k)=D[u / k] \eta$. Since $A_{\mathrm{cl}}$ is expressed in terms of it by eq.(16), this implies $\delta A_{\mathrm{cl}}=$ 
$\bar{D}\left[A_{\mathrm{cl}}\right] \eta$. If we make a simultaneous transformation on the integration variables, $\delta \alpha_{i}^{a}=f_{b c}^{a} \eta^{b} \alpha_{i}^{c}$, one finds that

$$
\delta \frac{1}{2} \int \alpha_{i}^{a} \frac{\partial^{2} \Gamma^{0}\left[A_{c l}\right]}{\partial A_{c l}^{a} \partial A_{c l}^{b}} \alpha_{i}^{b}=0 .
$$

This can be seen most easily by combining the transformations in $A=A_{\mathrm{cl}}+\alpha_{i}$ and using

$$
\delta\left(\Gamma^{0}[A]-\frac{1}{2 \pi x} \int \operatorname{tr}\{A u / k\}\right)=\frac{1}{2 \pi x} \int \operatorname{tr}\{\eta \bar{\partial}(u / k)\}
$$

The gauge variation of $W[u]$ is then obtained from an anomaly calculation. In the present setup, where jacobians for simultaneous transformation of original and PVfields cancel, this means that it is given by the expectation value of the variation of the PV-massterm (which is not invariant). We follow the method of 15]. Introducing a $T$-operator, by writing the mass term eq.(33) as

$$
\frac{1}{2} M_{i}^{2} \int \alpha_{i}^{a} T_{a b} \alpha_{i}^{b}
$$

one finds

$$
\delta W_{1 \text { loop }}\left[u\left(A_{\mathrm{cl}}\right)\right]=\sum_{i=1} c_{i} \operatorname{Tr}\left\{\eta\left(1-T^{-1} \Gamma^{0^{\prime \prime}} / M_{i}^{2}\right)^{-1}\right\}
$$

where Tr denotes a matrix trace (which we have explicitly taken to be in the adjoint representation) and a coordinatespace integration also.

To proceed, we recast the operator $T^{-1} \Gamma^{0^{\prime \prime}}$ in a more convenient form, using covariant derivatives and the operator identity eq.(18). We find

$$
T^{-1} \Gamma^{0^{\prime \prime}}=4\left(\bar{D}+A_{\mathrm{cl}}\right)^{2} \frac{D}{\bar{D}}
$$

where $D=D[u / k]$ and $\bar{D}=\bar{D}\left[A_{\mathrm{cl}}\right]$. It is important to realise that $D$ and $\bar{D}$ commute, due to the zero curvature condition (7). Therefore, they can be diagonalised simultaneously. To evaluate the trace, one can make use of this property by not using plane waves, but instead a basis of their eigenstates (i.e. $g e^{i k x}$ when $A_{\mathrm{cl}}=\bar{\partial} g g^{-1}$ ):

$$
\begin{aligned}
& \delta W_{1 \text { loop }}\left[u\left(A_{\mathrm{cl}}\right)\right] \\
& =\sum_{j=1} c_{j} \int_{0}^{\infty} d \lambda e^{-\lambda} \int d^{2} x \int \frac{d^{2} k}{(2 \pi)^{2}} \operatorname{tr}\left\{e^{-i k \cdot x} g^{-1} \eta e^{\left.\lambda T^{-1} \Gamma^{0^{\prime \prime}} / M_{j}^{2} g e^{+i k \cdot x}\right\}}\right. \\
& =\sum_{j=1} c_{j} \int_{0}^{\infty} d \lambda e^{-\lambda} \int d^{2} x \int \frac{d^{2} k}{(2 \pi)^{2}} \operatorname{tr}\left\{e^{-i k \cdot x} \eta_{g} e^{\lambda g^{-1} T^{-1} \Gamma^{0^{\prime \prime}} g / M_{j}^{2}} e^{+i k \cdot x}\right\}
\end{aligned}
$$


with

$$
\begin{aligned}
g^{-1} T^{-1} \Gamma^{0^{\prime \prime}} g & =4\left\{\partial \bar{\partial}+2 A_{\mathrm{cl} g} \partial+\left(\bar{\partial}\left(A_{\mathrm{cl} g}\right)+A_{\mathrm{cl} g}^{2}\right) \frac{\partial}{\bar{\partial}}\right\} \\
B_{g} & =g^{-1} B g \quad\left(B=\eta, A_{\mathrm{cl}}\right) .
\end{aligned}
$$

To evaluate eq.(40), we split the operator (41) into two pieces, viz. the second derivative $\partial \bar{\partial}$ and the rest, called $Y$ from now on. We follow the method used in 16 for differential operators, but extend here its use to a non-local differential operator. Applying the Baker-Campbell-Hausdorff formula, and rescaling the momenta, we find the following intermediate result

$$
\begin{aligned}
\delta W_{1 \text { loop }}\left[u\left(A_{\mathrm{cl}}\right)\right] \\
=\sum_{j=1} c_{j} \int_{0}^{\infty} d \lambda e^{-\lambda} \int d^{2} x \int d^{2} p_{j} \frac{M_{j}^{2}}{\lambda(2 \pi)^{2}} e^{-\left|p_{j}\right|^{2}} \\
\quad \operatorname{tr}\left\{\eta_{g} e^{-i M_{j} p_{j} \cdot x / \sqrt{\lambda}} e^{\left(4 \lambda Y / M_{j}^{2}+8 \lambda^{2}[\partial \bar{\partial}, Y] / M_{j}^{4}+\cdots\right)} e^{+i M_{j} p_{j} \cdot x / \sqrt{\lambda}}\right\} .
\end{aligned}
$$

The next step is to expand the exponential consisting of the non-local differential operators and let it act on the plane wave. In the limit $M_{j}^{2} \rightarrow \infty$, very few terms will contribute, but it is not immediately clear how to handle the non-locality. We have verified that the so-called pseudo-differential operator calculus (for a general reference, see [17]) works well. The Leibniz rule is still valid, when written in the form

$$
\partial^{n}(f *)=\sum_{k=0}^{\infty} \frac{n \ldots(n-k+1)}{k !}\left(\partial^{k} f\right) \partial^{n-k} * .
$$

If $n$ is positive the infinite sum just runs up to $k=n$. For $n$ negative however, there is an infinite number of terms. Whereas this might complicate matters, for the present purpose the series is effectively finite, due to the angular integral, combined with the $M_{j} \rightarrow \infty$ limit. After the angular integral only two pieces remain. In order $M_{j}^{2}$

$$
\begin{aligned}
& \delta_{1} W_{1 \text { loop }}\left[u\left(A_{\mathrm{cl}}\right)\right] \\
& =\sum_{j=1} c_{j} \int_{0}^{\infty} d \lambda e^{-\lambda} \int d^{2} x \int d^{2} p_{j} \frac{M_{j}^{2}}{\lambda(2 \pi)^{2}} \operatorname{tr}\{\eta\} e^{-\left|p_{j}\right|^{2}},
\end{aligned}
$$

which vanishes since $f_{a b}^{a}=0$. As a result the variation of the effective action contains no infinite terms. In order $M_{j}^{0}$,

$$
\begin{aligned}
& \delta_{[\partial \bar{\partial}, Y]} W_{1 \text { loop }}\left[u\left(A_{\mathrm{cl}}\right)\right] \\
& =\sum_{j=1} c_{j} \int_{0}^{\infty} d \lambda e^{-\lambda} \int d^{2} x \int d^{2} p_{j} \frac{2 \lambda}{\pi^{2} M_{j}^{2}} e^{-\left|p_{j}\right|^{2}}
\end{aligned}
$$




$$
\begin{aligned}
& \operatorname{tr}\left\{\eta_{g} e^{-i M_{j} p_{j} x / \sqrt{\lambda}}[\partial \bar{\partial}, Y] e^{+i M_{j} p_{j} x / \sqrt{\lambda}}\right\} \\
= & \frac{-1}{\pi} \sum_{j=1} c_{j} \int d^{2} x \operatorname{tr}\left\{\eta_{g} \partial\left(A_{\mathrm{cl} g}\right)\right\} \int d\left(\left|p_{j}\right|^{2}\right) e^{-\left|p_{j}\right|^{2}}\left|p_{j}\right|^{2} \\
= & \frac{1}{\pi} \int d^{2} x \operatorname{tr}\left\{\eta D[u / k] A_{\mathrm{cl}}\right\} .
\end{aligned}
$$

Rewriting it in a representation independent way this becomes

$$
\begin{aligned}
\delta W_{1 \text { loop }}\left[u\left(A_{\mathrm{cl}}\right)\right] & =\frac{-2 \tilde{h}}{2 \pi x} \int d^{2} x \operatorname{tr}\left\{A_{\mathrm{cl}} \delta(u / k)\right\} \\
& =2 \tilde{h} \int d^{2} x \frac{\partial W^{0}[u / k]}{\partial u^{a} / k} \delta\left(u^{a} / k\right) .
\end{aligned}
$$

Since there are no gauge invariant polynomials in terms of $u / k$ alone, we have proven the following one loop result:

$$
W[u]=(k+2 \tilde{h}) W^{0}\left[\frac{u}{k}\right]
$$

\section{Three derivations revisited}

Having completed our one-loop calculation in a regularised framework, we now reexamine the more heuristic methods reviewed in section three.

It is clear that result eq.(44) of our calculation, agrees with the KPZ-result eq. (27), that was based on invariance under vector-like transformations. The reason for this agreement is not so clear however. Indeed, the non-local regularisation we introduced, does not respect the vector-invariance explicitly. In particular, the gauge field measure is not invariant by itself. But neither is the ghost field measure. We have not specified how the ghost integral was regularised, but this is well known: one obtains the standard result in eq.(20) for the chiral determinant by regularising with a standard (non-invariant) massterm.

To check the KPZ method in the light of our treatment, one has to check that the quantum theory preserves the vector-invariance. This amounts to a check of quantum BRST invariance for eq. (23). It is well known how to do this. The BRST anomaly calculation splits into two distinct pieces. The ghost part calculation can most easily be read off from the result of the ghost determinant, and is therefore proportional to $2 \tilde{h} \delta W^{0}[\hat{\bar{A}}] / \delta \hat{\bar{A}} D[\hat{\bar{A}}] c$. For the gauge field part we follow the lines of [18. One then has to determine the expectation value of the variation of the PVmass term, just as in the calculation of section five. In fact, one is just repeating the steps of that section, with the gauge transformation parameter $\eta$ replaced by the BRST ghost $c$. The result can be read off from eq.(43), and exactly cancels the ghost contribution. This confirms that our gauge field loop regularisation, when combined with the usual ghost treatment, preserves the BRST invariance. 
Now we also make the connection with the semiclassical reasoning of section three, based on the factorisation of determinants. Although the operator equality eq.(18) is on solid enough ground, the statement about the determinants requires a closer look at regularisations. In section three, we used the standard values for the determinants of covariant derivatives, eq.(20). But from the present point of view, this means that certain regularisations -in fact those regularisations defined by the simplest possible massterms- are implicit. On the other hand the determinant of the non-local operator for the $A$-fields has been defined in its own right in the preceding section. We can then check whether the determinant formula of (19) agrees with these choices or not. We find that it should be replaced by

$$
\operatorname{det}_{(\mathrm{R})} \frac{\delta^{2} \Gamma^{0}\left[A_{\mathrm{cl}}\right]}{\delta A_{\mathrm{cl}} \delta A_{\mathrm{cl}}}=\frac{\operatorname{det}_{(\mathrm{S})} D[u / k]}{\operatorname{det}_{(\mathrm{S})} \bar{D}\left[A_{\mathrm{cl}}\right]} e^{-\frac{2 \tilde{h}}{2 \pi x} \int \operatorname{tr}\left\{A_{\mathrm{cl}} u / k\right\}}
$$

where we explicitly indicated our choice of regularisation for the non-local $A$ determinant by $(\mathrm{R})$, and the standard choices for the determinants of the covariant derivatives by $(\mathrm{S})$.

It may be interesting to note that there is an alternative way of writing the semiclassical determinant in a factorised form that does agree with our $A$-measure, viz.

$$
\operatorname{det}_{(\mathrm{R})} \frac{\delta^{2} \Gamma^{0}\left[A_{\mathrm{cl}}\right]}{\delta A_{\mathrm{cl}} \delta A_{\mathrm{cl}}}=\frac{\left(\operatorname{det}_{(\mathrm{S})} D[u / k]\right)^{2}}{\operatorname{det}_{(\mathrm{V})}\left\{\bar{D}\left[A_{\mathrm{cl}}\right] D[u / k]\right\}}
$$

where $(\mathrm{V})$ indicates a vector-invariant regularisation. The log of the determinant in the denominator is proportional to the covariant induced action of eq.(22) in terms of the chiral gauge fields $A_{\mathrm{cl}}$ and $u / k$. Since these are related to each other through the Legendre transform (16), that determinant equals unity. For the nonlocal determinant itself we conclude that, with the present definitions,

$$
\left(\operatorname{det}_{(\mathrm{R})} \frac{\delta^{2} \Gamma^{0}\left[A_{\mathrm{cl}}\right]}{\delta A_{\mathrm{cl}} \delta A_{\mathrm{cl}}}\right)^{\frac{1}{2}}=\operatorname{det}_{(\mathrm{S})} D[u / k]=e^{2 \tilde{h} W^{0}[u / k]}
$$

It may be noted that the determinant factorisation formula eq.(19) has been used quite often to calculate effective actions [10, 19, 4, 6, 20], almost always however in a rather formal way. Additional insight may be gained by looking back at the calculation of section four, when interpreting it in terms of these determinants. From the formal eq. (20), one would have expected the quadratic terms of the one loop contribution to the effective action to vanish. Rather, we found eq.(38). It was also pointed out after eq.(36) that a vanishing result would be obtained if one would regulate by inserting exponential cutoffs by hand. This explains why this last method gives a result that is the same as the one based on the straightforward determinant factorisation formula. 
The third derivation in section three was built on a change of variable including a Jacobian, using the Polyakov-Wiegmann formula, and assuming the Haar invariance of the measure. Since we do not intend to give an explicit prescription here for the regularisation of the $g$-integral (see however [21, 22]), we can not go into this issue in a direct way. As an alternative, we propose to use formula (29) to define the $g$-integration, by relating the so far unspecified measure $\mathcal{D} g$ to the measure $\mathcal{D} A$ we did construct before. T To emphasize the regularisation dependence, we rewrite it as

$$
\mathcal{D} A_{(\mathrm{R})}=\mathcal{D} g_{(\mathrm{R})} e^{2 \tilde{h} S^{+}[g]}
$$

We now analyse the transformation properties of the resulting measure. We proceed by computing the transformation properties of our well-defined $A$-measure under finite gauge transformations. This will teach us how the group measure itself behaves under left multiplication. We introduce the finite gauge transforms

$$
\begin{aligned}
A_{h} & =\bar{\partial} h h^{-1}+h A h^{-1} \\
\hat{\bar{A}}_{h} & =\partial h h^{-1}+h \hat{\bar{A}} h^{-1}
\end{aligned}
$$

Using the result (44), we find to the one-loop order that

$$
\begin{gathered}
e^{-(k+2 \tilde{h}) W^{0}\left[\hat{\bar{A}}_{h}\right]}=\int \mathcal{D} A_{h} e^{-k\left(\Gamma^{0}\left[A_{h}\right]-\frac{1}{2 \pi x} \int \operatorname{tr}\left\{A_{h} \hat{\bar{A}}_{h}\right\}\right)} \\
\quad=\int \mathcal{D} A \frac{\mathcal{D} A_{h}}{\mathcal{D} A} e^{-k\left(\Gamma^{0}[A]-\frac{1}{2 \pi x} \int \operatorname{tr}\{A \hat{\bar{A}}\}+W^{0}\left[\hat{\bar{A}}_{h}\right]-W^{0}[\hat{\bar{A}}]\right)} .
\end{gathered}
$$

On the other hand, due to the Polyakov-Wiegmann identity, we have that

$$
\begin{aligned}
& e^{-2 \tilde{h} W^{0}\left[\hat{\bar{A}}_{h}\right]}=e^{-2 \tilde{h}\left(W^{0}[\hat{\bar{A}}]+S^{-}[h]-\frac{1}{2 \pi x} \int \operatorname{tr}\left\{h^{-1} \bar{\partial} h \hat{\bar{A}}\right\}\right)} \\
& =\int \mathcal{D} A e^{-k\left(\Gamma^{0}[A]-\frac{1}{2 \pi x} \int \operatorname{tr}\left\{\left(A+\frac{2 \tilde{h}}{k} h^{-1} \bar{\partial} h\right) \hat{\bar{A}}\right\}-W^{0}[\hat{\bar{A}}]\right)-2 \tilde{h} S^{-}[h]}
\end{aligned}
$$

Combining eqs. (49) and (50) we find

$$
\begin{aligned}
& \int \mathcal{D} A e^{-k\left(\Gamma^{0}[A]-\frac{1}{2 \pi x} \int \operatorname{tr}\{A \hat{\bar{A}}\}\right)} \frac{\mathcal{D} A_{h}}{\mathcal{D} A} \\
& =\int \mathcal{D} A e^{-k\left(\Gamma^{0}\left[A-\frac{2 \tilde{h}}{k} h^{-1} \bar{\partial} h\right]-\frac{1}{2 \pi x} \int \operatorname{tr}\{A \hat{\bar{A}}\}\right)-2 \tilde{h} S^{-}(h)}
\end{aligned}
$$

\footnotetext{
${ }^{4}$ We do not follow 23 in effectively taking the square root of this expression. Such a procedure would lead to a coupling renormalisation factor $k+\tilde{h}$ instead of $k+2 \tilde{h}$.
} 
where we performed a shift on the quantum field in the second line, such that in both cases the source $\hat{\bar{A}}$ couples just to $A \equiv \bar{\partial} g g^{-1}$. This enables us to write down the following Jacobian

$$
\frac{\mathcal{D} A_{h(\mathrm{R})}}{\mathcal{D} A_{(\mathrm{R})}}=e^{-2 \tilde{h}\left(S^{-}[h]-\int \frac{\delta \Gamma^{0}[A]}{\delta A^{a}}\left(h^{-1} \bar{\partial} h\right)^{a}\right)} .
$$

We want to interpret this $k$-independent factor as a regulated Jacobian for the change of variables from $A$ to $A_{h}$. For consistency, it must obey the group structure for subsequent transformations; this can be checked by rewriting

$$
\begin{aligned}
\frac{\mathcal{D} A_{h(\mathrm{R})}}{\mathcal{D} A_{(\mathrm{R})}} & =e^{-2 \tilde{h}\left(S^{-}[h]-\frac{1}{2 \pi x} \int \operatorname{tr}\left\{\partial g g^{-1} h^{-1} \bar{\partial} h\right\}\right)} \\
& =e^{-2 \tilde{h}\left(S^{-}[h g]-S^{-}[g]\right)}
\end{aligned}
$$

Using eq.(48) this reduces to the following transformation property for the group integration:

$$
\frac{\mathcal{D}(h g)_{(\mathrm{R})}}{\mathcal{D} g_{(\mathrm{R})}}=e^{+M_{1}[h g]-M_{1}[g]}
$$

with

$$
\begin{aligned}
M_{1}[g] & =-2 \tilde{h}\left(S^{+}[g]+S^{-}[g]\right) \\
& =\frac{2 \tilde{h}}{2 \pi x} \int d^{2} z \operatorname{tr}\left\{\bar{\partial} g g^{-1} \partial g g^{-1}\right\} .
\end{aligned}
$$

Our measure $\mathcal{D} g_{(\mathrm{R})}$ is clearly not invariant, f but it differs from an invariant one merely by a local counterterm. Within the framework of the WZW model, it is then very natural to incorporate this counterterm $M_{1}[g]$ into the action precisely to restore Haar-invariance:

$$
\mathcal{D} g_{(\text {Haar })}=\mathcal{D} g_{(\mathrm{R})} \exp -M_{1}[g]
$$

We now propose to carry over this counterterm to the non-local theory as well. The resulting measure will carry the subscript (Haar), the one used until now will occasionally get the subscript $(\mathrm{R})$. Although, when expressed in terms of the field $A$ the counterterm $M_{1}$ will of course be non-local, it originates from a perfectly local expression in $g$, which seems to be an excellent criterion to characterise the admissible counterterms in the induced WZW model. We can then recalculate the

\footnotetext{
${ }^{5}$ The regularisation dependence of the Haar invariance of the measure was previously stressed by P. van Nieuwenhuizen.
} 
effective action $W_{\text {Haar }}[u]$ that one obtains when including this counterterm in the action:

$$
\begin{aligned}
& e^{-W_{\text {Haar }}[u]}=\int \mathcal{D} A_{(\text {Haar })} e^{-k\left(\Gamma^{0}[A]-\frac{1}{2 \pi x} \int \operatorname{tr}\{A u / k\}\right)} \\
& \quad=\int \mathcal{D} A_{(\mathrm{R})} e^{-k\left(\Gamma^{0}[A]-\frac{1}{2 \pi x} \int \operatorname{tr}\{A u / k\}\right)-M_{1}[g(A)]} \\
& \quad=\int \mathcal{D} A e^{-(k+2 \tilde{h})\left(\Gamma^{0}[A]-\frac{1}{2 \pi x} \int \operatorname{tr}\left\{A \frac{u}{k+2 \tilde{h}}\right\}\right)+2 \tilde{h} S^{-}[g(A)]} .
\end{aligned}
$$

Parametrising $\frac{u}{k+2 \hat{h}}$ as $\partial \bar{g} \bar{g}^{-1}$ and using the Polyakov-Wiegmann identity, this becomes

$$
\begin{aligned}
& =e^{-(k+2 \tilde{h}) W^{0}\left[\frac{u}{k+2 \tilde{h}}\right]} \int \mathcal{D} A e^{-(k+2 \tilde{h}) \Gamma^{0}\left[A_{\bar{g}^{-1}}\right]+2 \tilde{h} S^{-}[g(A)]} \\
& =e^{-(k+2 \tilde{h}) W^{0}\left[\frac{u}{k+2 \tilde{h}}\right]} \int \mathcal{D} A_{\bar{g}^{-1}} e^{-k \Gamma^{0}\left[A_{\bar{g}^{-1}}\right]-M_{1}\left[A_{\bar{g}^{-1}}\right]}
\end{aligned}
$$

where the last path integral is just a constant. As a result we have obtained agreement with formula (31) of section three.

\section{$7 \quad$ Beyond One Loop}

The previous sections only treated the one loop contributions. In the present section, we extend the analysis to an arbitrary number of loops. We reconsider the analysis of KPZ, starting from the covariant action

$$
\Gamma[A]+\bar{\Gamma}[\bar{A}]-\frac{k}{2 \pi x} \int \operatorname{tr}\{A \bar{A}\}
$$

Fixing the gauge as in section three, and performing the ghost integral, leads to a fixed value for $\bar{A}$ and a renormalisation of the $\bar{\Gamma}$ term, and we have to consider only the $A$-loops.

The theory may be considered to be an approximation to one where the matter system is strongly coupled to the gauge field. Apart from the induced action, one then has also a term describing the gauge field itself. Taking this to be the ordinary Yang-Mills action, it is immediately clear that the propagators for the gauge field acquire much better convergence properties. From the point of view of the induced action, the Yang-Mills term constitutes a higher order derivative term. This is reminiscent of the method used in [24] for ordinary gauge theories. Since we want 
to keep the vector gauge invariance, the fact that it is covariant is also most welcome. Let us analyse the divergences in the remaining theory. The action now is

$$
S[A, \hat{\bar{A}}]=\Gamma^{0}[A]-\frac{1}{2 \pi x} \int d^{2} x \operatorname{tr}\left\{A \hat{\bar{A}}+\frac{1}{4 \Lambda^{2}} F^{\mu \nu} F_{\mu \nu}\right\},
$$

where $\Lambda^{2}$ is supposedly a large parameter, that will be sent to infinity in the end. The Yang-Mills term generates new three and four point vertices. Explicitly,

$$
\begin{aligned}
\frac{1}{4 \Lambda^{2}} \operatorname{tr}\left\{F^{\mu \nu} F_{\mu \nu}\right\}= & \frac{-2}{\Lambda^{2}} \operatorname{tr}\left\{(\partial A)^{2}+(\bar{\partial} \hat{\bar{A}})^{2}+2 \hat{\bar{A}} \partial \bar{\partial} A\right. \\
& \left.+2[A, \hat{\bar{A}}]\left(\partial A-\bar{\partial} \hat{\bar{A}}+\frac{1}{2}[A, \hat{\bar{A}}]\right)\right\} .
\end{aligned}
$$

Expansion of the action around the same classical point $A_{\mathrm{cl}}$ as before (see eq.(16) with $u=k \hat{\bar{A}})$ yields

$$
\begin{aligned}
S\left[A_{\mathrm{cl}}+\alpha, \hat{\bar{A}}\right]= & W^{0}[\hat{\bar{A}}]+\frac{1}{2} \int \alpha^{a} \frac{\delta^{2} \Gamma^{0}\left[A_{\mathrm{cl}}\right]}{\delta A_{\mathrm{cl}}^{a} \delta A_{\mathrm{cl}}^{b}} \alpha^{b}+f\left(A_{\mathrm{cl}}\right) \alpha^{3}+\ldots \\
& -\frac{1}{\Lambda^{2} \pi x} \int \operatorname{tr}\left\{\alpha \partial^{2} \alpha+[\alpha, \hat{\bar{A}}]([\hat{\bar{A}}, \alpha]-2 \partial \alpha)\right\}
\end{aligned}
$$

where we used that $F^{\mu \nu}\left[A_{\mathrm{cl}}, \hat{\bar{A}}\right]=0$. The kinetic term for the quantum field $\alpha$ is

$$
\frac{1}{4 \pi x} \int \operatorname{tr}\left\{\alpha \frac{\partial}{\bar{\partial}}\left(1-\frac{4 \partial \bar{\partial}}{\Lambda^{2}}\right) \alpha\right\},
$$

leading to the improved UV behaviour of the Feynman diagrams. In fact the inclusion of the Yang-Mills term regularises all the diagrams made up with the original $\Gamma^{0}\left[A_{\mathrm{cl}}\right]$-vertices, as can be seen by calculating their superficial degree of divergence $(S D D)$. Each vertex adds to the integrand 2 powers of momenta minus the number of internal lines it couples to, or better. With $V$ the number of vertices and $I$ the number of internal lines, the total power contributed by the vertices is $2 V-2 I$ or less. The number of loops $L$ being equal to $I-V+1$, we have that $S D D \leq 2-2 I=4-2 L-2 V$. The tadpole or (higher order) seagull diagrams are in fact more convergent than this, because their vertex behaves as an inverse momentum. Inclusion of the extra Yang-Mills vertices modifies the formula to $S D D \leq 4-2 L-2 V_{\text {old }}-E_{\text {new }}$ where $V_{\text {old }}$ is the number of old vertices, and $E_{\text {new }}$ is the number of external lines coupling to the new vertices. There are only 2 ill-defined diagrams left in our theory. They are in fact topologically the same as in fig. 1 and 2, but now with only the new Yang-Mills vertices present. Since these are one-loop diagrams, we can regulate them them by adding Pauli-Villars fields with the action

$$
\begin{aligned}
S_{P V}\left[\alpha_{i}, A_{\mathrm{cl}}, \hat{\bar{A}}\right]= & \frac{1}{2} \int \alpha_{i}^{a} \frac{\delta^{2} \Gamma^{0}\left[A_{\mathrm{cl}}\right]}{\delta A_{\mathrm{cl}}^{a} \delta A_{\mathrm{cl}}^{b}} \alpha_{i}^{b} \\
& -\frac{1}{\Lambda^{2} \pi x} \int \operatorname{tr}\left\{\alpha_{i} \partial^{2} \alpha_{i}+\left[\alpha_{i}, \hat{\bar{A}}\right]\left(\left[\hat{\bar{A}}, \alpha_{i}\right]-2 \partial \alpha_{i}\right)\right\} \\
& -\frac{M_{i}^{2}}{16 \pi x} \int \operatorname{tr}\left\{\alpha_{i} \frac{1}{\bar{\partial}^{2}}\left(1-\frac{4 \partial \bar{\partial}}{\Lambda^{2}}\right) \alpha_{i}\right\} .
\end{aligned}
$$


This PV-action has some unfamiliar features. First, it may be noted that we have chosen a somewhat complicated looking mass term. This just serves to simplify the resulting Feynman diagrams (the PV propagators factorise easily with this choice: the denominator becomes $\left.\left(|q|^{2}+\Lambda^{2}\right)\left(|q|^{2}+M_{i}^{2}\right)\right)$, but is in fact immaterial: we have checked that all conclusions to be drawn below are valid also when one leaves out the $M^{2} / \Lambda^{2}$ terms. For $\Lambda \rightarrow \infty$ the mass term is of course the same as eq.(33). The second point is that the PV-terms only serve to regulate diagrams with external $\hat{\bar{A}}$ lines, not the propagating $\alpha$ lines. All loops with attached $\alpha$ lines were already made finite by the inclusion of the higher derivatives. For simplicity, we have therefore decoupled the PV fields from the quantum $\alpha$ fields. The coupling to the $A_{\mathrm{cl}}$ is kept for covariance. Presumably coupling PV-fields according to the general procedure of duplicating all couplings gives the same result, but we have not checked this explicitly. The decoupling has as a consequence that the PV-fields do not contribute to higher loop diagrams at all, not even to compensate divergent subgraphs, since according to the counting above there should not be any after the introduction of the Yang-Mills term: only a few diagrams with external $\hat{\bar{A}}$ and $A_{\mathrm{cl}}$ lines (which do not propagate) are involved in the additional $\mathrm{PV}$-regularisation.

Having modified the regularisation method for the purpose of rendering finite the more loop diagrams, it is important to check that the one loop results of sections four and five remain valid. A priori, they correspond to the present setup, where the $\Lambda \rightarrow \infty$ limit is taken first. However, it is not clear whether the order of limits matters. We will therefore recompute the 1-loop two point function, i.e. the quadratic part of $W\left[k \hat{\bar{A}}\left(A_{\mathrm{cl}}\right)\right]$.

The building blocks for our Feynman diagrams are

$$
\begin{aligned}
& \Delta_{a b}^{i}(q, r)=g_{a b} \delta^{2}(q+r) \frac{\left(|q|^{2}+\Lambda^{2}\right)\left(|q|^{2}+M_{i}^{2}\right)}{q^{2} \Lambda^{2}} \\
& \sum_{n=3}^{\infty} V_{a b}^{n}\left[A_{\mathrm{cl}}\right](q, r) \\
& \bar{V}_{a b}^{3}[\hat{\bar{A}}](q, r)=\frac{2 i}{\Lambda^{2}(2 \pi)^{2}} \int d^{2} p f_{a b c} \hat{\bar{A}}^{c}(p)(\bar{q}-\bar{r}) \delta^{2}(p+q+r) \\
& \bar{V}_{a b}^{4}[\hat{\bar{A}}](q, r)=\frac{-4}{\Lambda^{2}(2 \pi)^{4}} \int d^{2} p d^{2} s f_{a d}^{c} f_{c e b} \hat{\bar{A}}^{d}(p) \hat{\bar{A}}^{e}(s) \delta^{2}(p+q+r+s) .
\end{aligned}
$$

Note that partly they are expressed in terms of $A_{\mathrm{cl}}$ and partly in terms of $\hat{\bar{A}}$. For the present calculation it suffices to put $\hat{\bar{A}}(p)=\frac{\bar{p}}{p} A_{\text {cl }}(p)$, and one has to evaluate the following expression, consisting of 5 different diagrams (topologically, figs. 1 and 2 , but with both the old non-local vertices and the new Yang-Mills vertices):

$$
\begin{aligned}
& \frac{1}{2} \sum_{i} c_{i} \operatorname{tr}\left\{\Delta_{i}^{-1}\left(V^{4}+\bar{V}^{4}\right)\right\}-\frac{1}{4} \sum_{i} c_{i} \operatorname{tr}\left\{\Delta_{i}^{-1}\left(V^{3}+\bar{V}^{3}\right) \Delta_{i}^{-1}\left(V^{3}+\bar{V}^{3}\right)\right\} \\
& =-\frac{\tilde{h}}{(2 \pi)^{4}} \int d^{2} p A_{\mathrm{cl}}^{a}(p)\left(I_{4}+I_{\overline{4}}+I_{33}+2 I_{3 \overline{3}}+I_{\overline{3} \overline{3}}\right) A_{\mathrm{cl} a}(-p),
\end{aligned}
$$


where the notation reflects the vertices used, the overline denoting the Yang-Mills vertices. Let us start with the two diagrams where only the Yang-Mills vertices are present. The seagull graph vanishes

$$
I_{\overline{4}}=-\frac{2 \bar{p}^{2}}{p^{2}} \sum_{i} c_{i} \int d^{2} q \frac{q^{2}}{\left(|q|^{2}+\Lambda^{2}\right)\left(|q|^{2}+M_{i}^{2}\right)}=0
$$

due to the angular integral. The vacuum graph reads

$$
I_{\overline{3} \overline{3}}=\frac{\bar{p}^{2}}{p^{2}} \sum_{i} c_{i} \int d^{2} q \frac{q^{2}(p+q)^{2}(2 \bar{q}+\bar{p})^{2}}{\left(|q|^{2}+\Lambda^{2}\right)\left(|q|^{2}+M_{i}^{2}\right)\left(|p+q|^{2}+\Lambda^{2}\right)\left(|p+q|^{2}+M_{i}^{2}\right)} .
$$

The numerator is of order $q^{6}$, to start with, but the PV-contributions take care of the regularisation. Introducing Feynman parameters, shifting the momenta, and doing the angular integral, leads to

$$
\begin{aligned}
I_{\overline{3} \overline{3}}= & \sum_{i} c_{i} \frac{1}{\left(M_{i}^{2}-\Lambda^{2}\right)^{2}} \int_{0}^{1} d \alpha \int_{\Lambda^{2}}^{M_{i}^{2}} d x \int_{\Lambda^{2}}^{M_{i}^{2}} d y \\
& \int_{0}^{\infty} d\left(|q|^{2}\right) \frac{|q|^{4} A+|q|^{2} B+C}{\left\{|q|^{2}+(1-\alpha) x+\alpha y+\alpha(1-\alpha)|p|^{2}\right\}^{4}}
\end{aligned}
$$

where $A, B$ and $C$ depend only on $p, \bar{p}$ and $\alpha$. The numerator is now quartic in the momenta. It is then clear that $I_{\overline{3} \overline{3}}$ will become zero in the limit where $M_{i}^{2}$ and $\Lambda^{2}$ go to infinity. Thus, the extra diagrams with only Yang-mills couplings, which are the only ones having a divergence left before the introduction of PV-fields, give no contribution. This might have been guessed already from eqs. (56) and (57) without introducing the compensating PV-contributions. However, also in view of eq.(36), we resisted the temptation to apply formal simplifications to unregularised expressions.

We now proceed with the two "original" diagrams.

$$
\begin{aligned}
I_{4}+I_{33} \\
=\sum_{i} c_{i} \int d^{2} q \frac{\Lambda^{2}}{p^{2}} \frac{(\bar{p}+\bar{q})(p-q)(\bar{p} q-\bar{q} p)}{\left(|q|^{2}+\Lambda^{2}\right)\left(|q|^{2}+M_{i}^{2}\right)|p+q|^{2}} \\
+\int d^{2} q \frac{\Lambda^{4}}{p^{2}} \frac{(\bar{p} q-\bar{q} p)^{2}}{\left(|q|^{2}+\Lambda^{2}\right)\left(|q|^{2}+M_{i}^{2}\right)\left(|p+q|^{2}+\Lambda^{2}\right)\left(|p+q|^{2}+M_{i}^{2}\right)} \\
=\sum_{i} c_{i} \frac{2 \pi \Lambda^{2}}{\left(M_{i}^{2}-\Lambda^{2}\right)} \frac{|p|^{2}}{p^{2}} \int_{0}^{1} d \alpha \int_{\Lambda_{i}^{2}}^{M_{i}^{2}} d x \frac{(1-\alpha)}{\left\{(1-\alpha) x+\alpha(1-\alpha)|p|^{2}\right\}} \\
-\frac{2 \pi \Lambda^{4}}{\left(M_{i}^{2}-\Lambda^{2}\right)^{2}} \frac{|p|^{2}}{p^{2}} \int_{0}^{1} d \alpha \int_{\Lambda^{2}}^{M_{i}^{2}} d x \int_{\Lambda^{2}}^{M_{i}^{2}} d y \frac{\alpha(1-\alpha)}{\left\{(1-\alpha) x+\alpha y+\alpha(1-\alpha)|p|^{2}\right\}^{2}}
\end{aligned}
$$


Here it matters in which order the regulators are removed. If the Pauli-Villars masses go to infinity, $\Lambda^{2}$ remaining finite, the Pauli-Villars terms drop out, and

$$
\begin{aligned}
\lim _{\Lambda \rightarrow \infty} \lim _{M_{i} \rightarrow \infty}\left(I_{4}+I_{33}\right) & =\frac{-2 \pi|p|^{2}}{p^{2}} \lim \int_{0}^{1} d \alpha \ln \frac{\alpha \Lambda^{2}+\alpha(1-\alpha)|p|^{2}}{\Lambda^{2}+\alpha(1-\alpha)|p|^{2}} \\
& =\frac{2 \pi|p|^{2}}{p^{2}}
\end{aligned}
$$

In the opposite order one finds that

$$
\begin{aligned}
\lim _{M_{i} \rightarrow \infty} \lim _{\Lambda \rightarrow \infty}\left(I_{4}+I_{33}\right) & =\lim \sum_{i} c_{i} \frac{-2 \pi|p|^{2}}{p^{2}} \int_{0}^{1} d \alpha \ln \frac{\alpha M_{i}^{2}+\alpha(1-\alpha)|p|^{2}}{M_{i}^{2}+\alpha(1-\alpha)|p|^{2}} \\
& =\frac{-2 \pi|p|^{2}}{p^{2}}
\end{aligned}
$$

which is of course the result eq.(37) that we obtained in section four without introducing $\Lambda$ in the first place.

The remaining contribution equals

$$
\begin{aligned}
2 I_{3 \overline{3}} & =\sum_{i} c_{i} \int d^{2} q \frac{2 \Lambda^{2}}{p^{2}} \frac{(\bar{p} q-\bar{q} p) q \bar{p}(2 \bar{q}+\bar{p})(p+q)}{\left(|q|^{2}+\Lambda^{2}\right)\left(|q|^{2}+M_{i}^{2}\right)\left(|p+q|^{2}+\Lambda^{2}\right)\left(|p+q|^{2}+M_{i}^{2}\right)} \\
= & \sum_{i} c_{i} \frac{-8 \pi \Lambda^{2}}{\left(M_{i}^{2}-\Lambda^{2}\right)^{2}} \frac{|p|^{2}}{p^{2}} \int_{0}^{1} d \alpha \int_{\Lambda^{2}}^{M_{i}^{2}} d x \int_{\Lambda^{2}}^{M_{i}^{2}} d y \frac{\alpha(1-\alpha)}{\left\{(1-\alpha) x+\alpha y+\alpha(1-\alpha)|p|^{2}\right\}} \\
& -\frac{4 \pi \Lambda^{2}}{\left(M_{i}^{2}-\Lambda^{2}\right)^{2}} \frac{|p|^{4}}{p^{2}} \int_{0}^{1} d \alpha \int_{\Lambda^{2}}^{M_{i}^{2}} d x \int_{\Lambda^{2}}^{M_{i}^{2}} d y \frac{\alpha^{3}(1-\alpha)}{\left\{(1-\alpha) x+\alpha y+\alpha(1-\alpha)|p|^{2}\right\}^{2}} .
\end{aligned}
$$

The second term is equal to zero, no matter how the limits are taken. The rest gives

$$
\begin{aligned}
\lim _{\Lambda \rightarrow \infty} \lim _{M_{i} \rightarrow \infty} 2 I_{3 \overline{3}} & =\lim -\frac{8 \pi|p|^{2}}{p^{2}} \int_{0}^{1} d \alpha \ln \Lambda^{2}-\alpha \ln \left(\alpha \Lambda^{2}\right)-(1-\alpha) \ln \left((1-\alpha) \Lambda^{2}\right) \\
& =-\frac{4 \pi|p|^{2}}{p^{2}}
\end{aligned}
$$

and

$$
\begin{aligned}
\lim _{M_{i} \rightarrow \infty} \lim _{\Lambda \rightarrow \infty} 2 I_{3 \overline{3}} & =\frac{16 \pi|p|^{2}}{p^{2}} \sum_{i} c_{i} \int_{0}^{1} d \alpha \alpha \ln \alpha \\
& =0 .
\end{aligned}
$$

Summing all five diagrams we have, for both limits,

$$
W_{\text {quantum }}\left[k \hat{\bar{A}}\left(A_{\mathrm{cl}}\right)\right]=-\frac{\tilde{h}}{2 \pi x} \int d^{2} x \operatorname{tr}\left\{A_{\mathrm{cl}} \frac{\partial}{\partial} A_{\mathrm{cl}}+\cdots\right\} .
$$

We have checked that all other $\Lambda, M_{i} \rightarrow \infty$ limits give the same answer too. It is satisfying that the sum of all one loop diagrams does not depend on the specific way 
of taking the limits, despite the fact that expressions for individual diagrams do. We conclude that the one loop results of section four remain valid.

To extend these result to all loops, we now continue along the line of section five, and compute the full quantum effective action through its (anomalous) gauge variation. Again, using the standard ghost determinant, we only have to calculate the gauge field contribution, with the aim to show that it cancels the ghost anomaly.

Since the regulating Yang-Mills term is invariant under the simultaneous transformation of classical and quantum fields, the Pauli-Villars mass term is still the only symmetry breaking part of the Lagrangian. The variation of $W_{\text {quantum }}[k \hat{\bar{A}}]$ can thus be written as the expectationvalue of the variation of the mass term of eq. (55). When expanding this expectationvalue in a series of Feynman diagrams, it can be seen that the only contributing diagrams are those with one loop of P.V. fields (and no other loops).

The full variation of $W_{\text {quantum }}[k \hat{\bar{A}}]$ is therefore given by

$$
\delta W_{\text {quantum }}[k \hat{\bar{A}}]=\frac{1}{2} \sum_{j=1} c_{j} M_{j}^{2} \operatorname{Tr}\left\{X\left(\Delta^{j}+\sum_{n=3}\left(V^{n}\left[A_{\mathrm{cl}}(\hat{\bar{A}})\right]+\bar{V}^{n}[\hat{\bar{A}}]\right)\right)^{-1}\right\},
$$

where $X$ results from varying the mass term:

$$
X_{a b}(q, r)=\frac{1}{(2 \pi)^{2}} \int d^{2} p f_{a b c} \eta^{c}(p)\left\{\frac{1}{r^{2}}\left(\frac{\Lambda^{2}+|r|^{2}}{\Lambda^{2}}\right)-\frac{1}{q^{2}}\left(\frac{\Lambda^{2}+|q|^{2}}{\Lambda^{2}}\right)\right\} \delta^{2}(p+q+r) \text {. }
$$

The term without vertex vanishes because $\operatorname{tr}\{\eta\}$ is 0 . The next term is

$$
\begin{aligned}
\delta_{\overline{3}} W_{\text {quantum }}[k \hat{\bar{A}}] & =-\frac{1}{2} \sum_{j=1} c_{j} M_{j}^{2} \operatorname{Tr}\left\{X \Delta_{j}^{-1} \bar{V}^{3}[\hat{\bar{A}}] \Delta_{j}^{-1}\right\} \\
& =-\frac{\tilde{h}}{(2 \pi)^{4}} \int d^{2} p \hat{\bar{A}}^{a}(p) J_{\overline{3}} \eta_{a}(-p)
\end{aligned}
$$

with

$$
\begin{aligned}
J_{\overline{3}} & =\sum_{j=1} c_{j} M_{j}^{2} \int d^{2} q \frac{2 i q^{2}(\bar{p}+2 \bar{q})}{\left(|q|^{2}+\Lambda^{2}\right)\left(|q|^{2}+M_{j}^{2}\right)\left(|p+q|^{2}+M_{j}^{2}\right)} \\
& =\sum_{j=1} c_{j} \frac{-8 \pi i p M_{j}^{2}}{\left(M_{j}^{2}-\Lambda^{2}\right)} \int_{0}^{1} d \alpha \int_{\Lambda^{2}}^{M_{j}^{2}} d x \frac{\alpha(1-\alpha)}{\left\{(1-\alpha) x+\alpha M_{j}^{2}+\alpha(1-\alpha)|p|^{2}\right\}} .
\end{aligned}
$$

In the beginning of this section we demonstrated that the order of limits was immaterial for the sum of all contributions. Assuming that this extends to the present case, we evaluate

$$
\begin{aligned}
\lim _{\Lambda \rightarrow \infty} \lim _{M_{j} \rightarrow \infty} J_{\overline{3}} & =-8 \pi i p \lim \sum_{j=1} c_{j} \int_{0}^{1} d \alpha \alpha \ln \frac{M_{j}^{2}+\alpha(1-\alpha)|p|^{2}}{\alpha M_{j}^{2}+(1-\alpha) \Lambda^{2}+\alpha(1-\alpha)|p|^{2}} \\
& =2 \pi i p,
\end{aligned}
$$


leading to

$$
\begin{aligned}
\delta_{\overline{3}} W_{\text {quantum }}[k \hat{\bar{A}}] & =\frac{2 \tilde{h}}{2 \pi x} \int d^{2} x \operatorname{tr}\{\eta \bar{\partial} \hat{\bar{A}}\} \\
& =\delta\left(2 \tilde{h} W^{0}[\hat{\bar{A}}]\right) .
\end{aligned}
$$

Remarkably, with this order of limits, this single diagram already furnishes the entire quantum variation we expected to obtain. It remains to show that all the other graphs vanish.

As an illustration we compute the first term explicitly:

$$
\begin{aligned}
\delta_{3} W_{\text {quantum }}[k \hat{\bar{A}}] & =-\frac{1}{2} \sum_{j=1} c_{j} M_{j}^{2} \operatorname{Tr}\left\{X \Delta_{j}^{-1} V^{3}\left[A_{\mathrm{cl}}(\hat{\bar{A}})\right] \Delta_{j}^{-1}\right\} \\
& =-\frac{\tilde{h}}{(2 \pi)^{4}} \int d^{2} p A_{\mathrm{cl}}^{a}(p) J_{3} \eta_{a}(-p)
\end{aligned}
$$

with

$$
\begin{aligned}
J_{3} & =\sum_{j=1} c_{j} M_{j}^{2} \int d^{2} q \frac{\Lambda^{2} i(\bar{p} q-\bar{q} p)^{2}}{p|q|^{2}\left(|q|^{2}+M_{j}^{2}\right)\left(|p+q|^{2}+\Lambda^{2}\right)\left(|p+q|^{2}+M_{j}^{2}\right)} \\
& =\sum_{j=1} c_{j} \frac{-2 \pi i \bar{p} \Lambda^{2}}{\left(M_{j}^{2}-\Lambda^{2}\right)} \int_{0}^{1} d \alpha \int_{0}^{M_{j}^{2}} d x \int_{\Lambda^{2}}^{M_{j}^{2}} d y \frac{\alpha(1-\alpha)}{\left\{(1-\alpha) x+\alpha y+\alpha(1-\alpha)|p|^{2}\right\}^{2}}
\end{aligned}
$$

In the last line, there is a factor $M^{-2}$ multiplying an integral that diverges only logarithmically when $M \rightarrow \infty$. This can already be seen from the first line of eq. (64), by neglecting the momentum dependence of the two factors $M_{j}^{2}+|k|^{2}$, and counting the degree of divergence of the remaining $q$-integral. Consequently,

$$
\lim _{\Lambda \rightarrow \infty} \lim _{M_{j} \rightarrow \infty} J_{3}=0
$$

The same argument can be repeated for the diagrams with more and/or other insertions. Again, by moving in front two factors $M^{-2}$ and counting the degree of divergence of the remaining momentum integral, one finds at most logarithmic divergences, except in a few cases. Of these, one was computed in eq.(62). The other two have one $\bar{V}^{4}$ or two $\bar{V}^{3}$ insertions, and vanish also, by an explicit check. Consequently, with this order of the limits, eq. (63) gives indeed the total contribution.

If we check what happens when we again reverse the limits, we have

$$
\begin{aligned}
\lim _{M_{j} \rightarrow \infty} \lim _{\Lambda \rightarrow \infty} J_{3} & =-2 \pi i \bar{p} \sum_{j=1} c_{j} \int_{0}^{1} d \alpha \ln \frac{M_{j}^{2}+\alpha(1-\alpha)|p|^{2}}{\alpha M_{j}^{2}+\alpha(1-\alpha)|p|^{2}} \\
& =2 \pi i \bar{p},
\end{aligned}
$$

while the $\frac{1}{\Lambda^{2}}$ of the $\bar{V}^{3}[\hat{\bar{A}}]$ vertex makes $J_{\overline{3}}=0$ in this limit (see eq. (61)). Using the relation between $A_{\mathrm{cl}}$ and $\hat{\bar{A}}$, which to the first order is $\bar{p} A_{\mathrm{cl}}(p)=p \hat{\bar{A}}(p)$, we recover 
the full quantumvariation of eq.(63), plus additional terms with higher powers of the field $\hat{\bar{A}}$. These additional terms only drop out when including the contributions to eq. (59) of the P.V. diagrams with more $V^{n}\left[A_{\mathrm{cl}}\right]$ vertex insertions. These diagrams indeed give finite results in the limit we are considering 0 , such that the complete expression will emerge this time in the form of an infinite series

$$
\delta\left(2 \tilde{h} W^{0}\left[\hat{\bar{A}}\left(A_{\mathrm{cl}}\right)\right]\right)=\frac{2 \tilde{h}}{2 \pi x} \int d^{2} x \operatorname{tr}\left\{\eta D\left[-2 \pi \frac{\delta \Gamma^{0}\left[A_{\mathrm{cl}}\right]}{\delta A_{\mathrm{cl}}}\right] A_{\mathrm{cl}}\right\}
$$

In order to calculate the full effective action, it thus suffices to study its 1-loop variation. This 1-loop variation can be obtained in two ways: either as an infinite sum of diagrams made up with the 'original' non-local vertices; or by using a different order of limits, in which case all the information collapses in a single diagram.

\section{Conclusions}

Especially for two-dimensional theories, there has been a marked increase in the interest for non-local field theories, mainly when arising as induced theories describing the dynamics of degrees of freedom that arise through anomalies. Whereas for local field theories experience has accumulated considerably over the years, for non-local field theories a lot remains to be learned. In this paper we have shown that, as far as regularisation is concerned, one can go a long way with rather conventional methods. We have shown that Pauli-Villars methods can be extended quite easily, provided one is willing to introduce non-local mass terms. Also the method of higher covariant derivatives has proven useful in limiting the breaking of symmetries to a (presumably unavoidable) core. We have also shown that anomaly-style calculations have an equally enlarged field of application, and here also some extension of traditional methods (we used pseudo-differential operators) was needed. We have proposed a scheme for a specific class of non-local induced actions, viz. gauge field actions induced through coupling with affine currents, although without any doubt it can be extended to a large class of similar actions. We have used our scheme to clear up some discrepancies between different calculations of the (finite) renormalisation factors that allow one to express the effective quantum actions of these theories in terms of their classical actions. The conclusion here is, that different counterterms do indeed lead to different field renormalisation factors, but no inconsistency arises if one adopts a given regularisation procedure systematically — although it may not be obvious how to guarantee this in practice. We have shown the precise relation between different formal evaluations, in particular how different symmetry requirements, vector gauge symmetry conservation and Haar invariance, are related through a counterterm, that is local in the framework of a WZW lagrangian. It may be noted here that there exist other methods where the regularisation is much more implicit, as for example in point splitting methods combined with operator product

\footnotetext{
${ }^{6}$ In fact we already calculated these diagrams in section 5, albeit in configurationspace.
} 
expansions. We have not established a direct connection between that method and the regularisation(s) in this paper, but expect that there should be no objection to it either, if adopted systematically. We remind the reader that it leads to results 'halfway' between the vector-invariant and the Haar-invariant results.

One attempt to bring these non-local theories back to the more familiar local ground would be through a transformation of variables. For example, for induced gravity the Polyakov variables make the action local. The resulting local action however is rather involved, and the application of standard perturbative renormalisation theory is not straightforward. Since universal normalisation requirements for the resulting theories are lacking, we have been guided by the symmetry preservation of the theory, and have adopted the working rule not to add any counterterms unless required by symmetry. One can arrive at the same rule when considering the theory treated in section seven. The non-local action can be considered as arising from a local matter action, where one also includes the Yang-Mills term from the start. The renormalisation conditions and counterterms for this local field theory then will not involve the non-local vertices of the induced theory either. One has noticed (see section four) that we never needed to introduce counterterms to cancel infinities. The same property also invites one to perform formal calculations without even mentioning the divergences, although we have seen that the finite results obtained in this way may be incorrect. Physically, it is linked to the induced nature of the theories we considered (which may be the only non-local theories that one wants to consider anyway), which leads to much softer ultraviolet behaviour than expected generically.

Acknowledgements: We would like to thank Jan de Boer, Jose Figueroa, Toine Van Proeyen, A. Tseytlin and especially Peter van Nieuwenhuizen and Bernard de Wit for discussions.

\section{References}

[1] J.Wess, B.Zumino, Phys. Lett. B37(1971)95.

E. Witten, Comm. Math. Phys. 92 (1984) 455.

[2] A. M. Polyakov, in 'Fields, Strings and Critical Phenomena', eds. E. Brézin and J. Zinn-Justin, North-Holland (1990) (Les Houches 1988)

[3] V.G. Knizhnik, A.M. Polyakov and A.B. Zamolodchikov, Mod. Phys. Lett. A3 (1988) 819.

[4] K. Schoutens, A. Sevrin and P. van Nieuwenhuizen, Nucl. Phys. B371 (1992) 315.

[5] J. de Boer and J. Goeree, Utrecht preprint THU-92/33

J. de Boer, Doctoraatsthesis, Utrecht 1993. 
[6] A. Sevrin, K. Thielemans and W. Troost, LBL-33738, UCB-PTH-93/06, KULTF-93/09.

A. Sevrin and W. Troost, LBL-34125, UCB-PTH-93/19, KUL-TF-93/21.

[7] V. G. Drinfeld and V. V. Sokolov, J. Sov. Math. 30 (1984) 1975

L. Feher, L. O'Raifeartaigh, O. Ruelle, I. Tsutsui and A. Wipf, Phys. Rep. 222 (1992) 1.

[8] W. Pauli and P. Villars, Rev. Mod. Phys. 21 (1949) 434.

[9] A. M. Polyakov and P. B. Wiegmann, Phys. Lett. 131B (1983) 121; Phys. Lett. 141B (1984) 223.

[10] Al.B. Zamolodchikov, preprint ITEP $84-89$ (1989).

[11] K. Schoutens, A. Sevrin and P. van Nieuwenhuizen, in the proceedings of the Stony Brook conference Strings and Symmetries 1991, (World Scientific, 1992).

[12] O. Alvarez, Nucl. Phys. B238 (1984) 61

[13] M. T. Grisaru and P. van Nieuwenhuizen, Int. Jour. Mod. Phys. A7 (1992) 5691.

[14] F. De Jonghe, R. Siebelink, W. Troost, Phys. Lett. B288 (1992) 47.

[15] A. Diaz, W. Troost, P. van Nieuwenhuizen and A. Van Proeyen, Int. Jour. Mod. Phys. A4 (1989) 3959.

[16] P. Bouwknegt and P. van Nieuwenhuizen, Class. Quantum Gravity 3 (1986) 207

[17] M. A. Shuba, "Pseudodifferential Operators and Spectral Theory", Springer Verlag 1987.

[18] W. Troost, P. van Nieuwenhuizen and A. Van Proeyen, Nucl. Phys. B333 (1990) 727.

[19] K.A. Meissner and J. Pawełczyk, Mod. Phys. Lett. A5 (1990) 763.

[20] G.W. Delius, M.T. Grisaru and P. van Nieuwenhuizen, preprint CERNTH.6458/92.

[21] M. Bos, Phys. Lett. B189 (1987) 435

H. Leutwyler and M. Shifman, Int. Jour. Mod. Phys. A7 (1992) 795

[22] M. T. Grisaru, B. de Wit and P. van Nieuwenhuizen, Brandeis-Stony BrookUtrecht preprint.

[23] A. Tseytlin, Imperial college preprint 12/92 and CERN-TH.6804/93. 
[24] L. D. Faddeev and A. A. Slavnov, Gauge Fields: Introduction to Quantum Theory, Frontiers in Physics series 50, The Benjamin/Cummings Publishing Company, Reading, Massachussetts (1980). 